einer zweiter Art einen Schritt nach rechts). Man überzeugt sich leicht davon, daß $x$ zum Simplex $w$ gehört. Damit ist bewiesen, daß die $w$ das $\left(t_{\mu} ; t_{\nu}\right)$ ausfüllen.

12. Endlich wäre noch zu rechtfertigen, daß wir in die Zerlegungsformel unseres Satzes $w_{0}$ mit positivem Vorzeichen aufgenommen haben. Eine einfache Determinantenbetrachtung, die wir nicht ausführen, zeigt, daß, wenn $t_{\mu}$ und $t_{\nu}$ positives Volumen haben, auch $w_{0}$, also auch $\left(t_{u} ; t_{v}\right)$ positives Volumen hat.

Damit ist unser Satz vollständig bewiesen.

\section{Die Bettischen Gruppen der Verbindung zweier Polytope.}

\author{
Von
}

\section{Hans Freudenthal (Amsterdam).}

1. Unter der Verbindung $R \circ S$ zweier topologischer Räume $R$ und $S$ verstehen wir anschaulich die Vereinigung der Verbindungslinien je zweier Punkte $a \in R, b \in S$; dabei hat man sich $R$ und $S$ so vorzustellen, daß sich je zwei Verbindungslinien in "allgemeiner" Lage befinden, also nicht mehr gemein haben als evtl. Anfangsoder Endpunkt.

Exakt: Die Punkte von $R \circ S$ sind gewisse abgeschlossene Teilmengen des Cartesischen Produktes $R \times S \times L$ (hier bedeute $L$ die Strecke $0 \leqslant t \leqslant 1$ ), nämlich: $a \times S \times 0$ für $a \in R ; R \times b \times 1$ für $b \in S$, $a \times b \times t$ für $a \in R, b \in S, 0<t<1$. $R \circ S$ wird so topologisiert, wie das bei einer solchen Menge abgeschlossener Teilmengen üblich ist. Ist $R$ bzw. $S$ leer, so verstehe man unter $R \circ S$ den Raum $S$ bzw. $R$.

Sind $R$ und $S$ endliche Polytope ${ }^{1}$ ), so läßt sich $R \circ S$ rein kombinatorisch definieren: Simplexe

$$
t_{\rho} \circ u_{\sigma}=\left[a_{0} \ldots a_{\varrho} b_{0} \ldots b_{\sigma}\right]
$$

von $R \circ S$ sind die und nur die Eckpunktmengen, für die $t_{\rho}=\left[a_{0} \ldots a_{\varrho}\right]$ Simplex von $R$ und $\left[b_{0} \ldots b_{\sigma}\right]$ Simplex von $S$ ist. (Man beachte, da $\beta$ auch die leere Menge zu den Simplexen gehört.) Die Dimension von $R \circ S$ ist hier um Eins größer als die Summe der Dimensionen von $R$ und $S$.

1) Die Verbindung zweier Polytope ist ein bekanntes Hilfsmittel der Mannigfaltigkeitstheorie.

Fundamenta Mathematicae. T. Xxix. 
2. Bei festem $S$ sind natürlich z. B. die Bettischen, Gruppen von $R \circ S$ topologische Invarianten von $R$. In der Hoffnung, so neue topologische Invarianten eines Kompaktums $R$ zu finden, sieht man. sich aber getäuscht, da gegen alle Erwartung auch von $R \circ S$ (wie schon bekanntlich von $R \times S$ ) gilt, daß seine Bettischen Gruppen durch die von $R$ und $S$ vollständig bestimmt sind. Wir beschränken uns im Folgenden auf Polytope $R$ und $S$, da die Ausdehnung auf beliebige kompakte $R$ und $S$ nach wohlbekannten Verfahren erfolgen kann. Als Koeffizientenbereich legen wir den der ganzen Zahlen zugrunde.

$B_{0}$ bzw. $C_{\rho}$ bzw. $(B \times C)_{e}$ bzw. $(B \circ C)_{0}$ sei die $\varrho$-te Bettische Gruppe von $R$ bzw. $S$ bzw. $R \times S$ bzw. $R \circ S$. Win angehängter Stern bezeichne die zugehörige Torsionsgruppe.

Für das cartesische Produkt gilt dann die

$$
\text { Formel: } \quad(B \times C)_{\tau}=\sum_{\rho+\sigma=\tau} B_{\rho} C_{\sigma}+\sum_{\rho+\sigma=\tau-1} B_{\ell}^{*} C_{\sigma}^{*} .
$$

Summe und Produkt sind direlkt ${ }^{2}$ ) zu nehmen; die konkrete Bedeutung der direkten Summe und der direkten Produkte im ersten Summanden liegt dabei auf der Hand; die direkten. Produkte im zweiten Summanden lassen sich aber konkret nicht so einfach interpretieren.

Für die Verbindung hat man fast dieselbe

$$
\text { Formel: } \quad(B \circ C)_{\tau}=\sum_{\varrho+\sigma+1=\tau} B_{\varrho} C_{\sigma}+\sum_{\varrho+\sigma+1=\tau-1} B_{\varrho}^{*} C_{\sigma}^{*} ;
$$

doch muß man, wo es sich um die Verbindung handelt, immer auch mit Bettischen Gruppen (-1)-ter Dimension rechnen (die aus einem Zyklus der Ordnung 0 bestehen) und die 0-ten Bettischen Gruppen etwas modifizieren (so daß eine Erzeugende wegfällt). Diese Abän-

2) Für Gruppen von endlich vielen Erzeugenden (mit denen wir es hier nur zu tun haben) läßt sich das direkte Produkt durch die zwei Forderungen definieren:

1) es verhält sich distributiv zur direkten Summe,

2) es erzeugt aus zwei zyklhschen Gruppen der Ordnungen $p$ and $q$ eine der Ordnung $(p, q)$ (größter gemeinsamer Teiler von $p$ und $q$; dabei gilt 0 als Vielfaches aller Zahlen). derung geschieht am natürlichsten so $\left.^{3}\right)$ : (-1)-dimensionale Komplexe und Zyklen werden die Vielfachen des leeren Simplexes, Rand. eines 0-dimensionalen wird das leere Simplex.

Beide Formeln und ihre Beweise lassen sich in ein formales Schema einordnen. Der Beweis wird im Wesentlichen derselbe wie der für das Cartesische Produkt bei Alexandroff-Hopf ${ }^{3 a}$ ).

3. $k_{0}$ bzw. $l_{\sigma}$ mögen Linearformen der $t_{0}$ bzw. $u_{\sigma}$ bezeichnen; $k_{Q} \times l_{\sigma}$ bzw. $k_{Q} \circ l_{\sigma}$ wird in naheliegender Weise distributiv aus den $t_{Q} \times u_{\sigma}$ bzw. $t_{\ell} \circ u_{\sigma}$ zusammengesetzt. Ist $\mathfrak{r}$ der Randoperator, so hat man

also auch

$$
\begin{aligned}
& \mathfrak{r}\left(t_{0} \times u_{\sigma}\right)=\mathfrak{r} t_{0} \times u_{\sigma}-(-1)^{\varrho} t_{0} \times \mathfrak{r} u_{\sigma}, \quad 3 \mathrm{~b} \\
& \mathfrak{r}\left(t_{\sigma} \circ u_{\sigma}\right)=\mathfrak{r} t_{\varrho} \circ u_{\sigma}-(-1)^{\rho+1} t_{\rho} \circ \mathfrak{r} u_{\sigma}, \\
& \mathfrak{r}\left(k_{0} \times l_{\sigma}\right)=\mathfrak{r} k_{Q} \times l_{\sigma}-(-1)^{\circ} k_{Q} \times \mathfrak{r} l_{\sigma}, \\
& \mathfrak{r}\left(k_{\ell} \circ l_{\sigma}\right)=\mathfrak{r} k_{\ell} \circ l_{\sigma}-(-1)^{0+1} k_{\ell} \circ \mathrm{r} l_{\sigma} .
\end{aligned}
$$

Wir können (etwas allgemeiner) auch ausgehen von einem System $K$ additiver abelscher Gruppen $K_{\ell}, \varrho=-1,0, \ldots, d$; von denen jede in die vorangehende homomorph abgebildet ist, $\mathfrak{r} K_{0+1} \subset K_{\rho}$, $\mathfrak{r}^{2}=0$, und von einem ebensolchen System $L$ von abelschen Gruppen $L_{\sigma}$, $\left.\sigma=-1,0, \ldots, e .{ }^{4}\right)$ Wir definieren dann das System $K \cdot L$ :

ferner für $k_{\ell} \in K_{\ell}, l_{\sigma} \in L_{\sigma}$

$$
(K \cdot L)_{\tau}=\sum_{e+\sigma=\tau+1} K_{\ell} \cdot L_{\sigma}
$$

$$
\mathfrak{r}\left(k_{\varrho} \cdot l_{\sigma}\right)=\mathfrak{r} k_{\varrho} \cdot l_{\sigma}-(-1)^{\rho+1} k_{\varrho} \cdot r l_{\rho} .
$$

Man sieht leicht ein, daß auch in $K \cdot I$ gilt: $\mathrm{r}^{2}=0$. Auf Grund davon kann man nicht nur in $K$ und $L$, sondern auch in $K \cdot L$ „Bettische Gruppen" definieren, deren gegenseitiges Verhältnis durch die zweite der zu beweisenden Formeln beschrieben wird.

3) In dieser Form ist die Modifikation gelegentlich von Herrn D. van Dantzig vorgeschlagen worden. Die Modifikation erweist sich überall da als zweckmäßig, wo man - wie bei der Verbindung - das leere Simplex zulassen muß; dagegen verdienen die gewöhnlichen Bettischen Gruppen überall da den Vorzug, wo - wie beim Cartesischen Produkt - man das leere Simplex zu vermeiden hat.

8a) Topologie (1935), 308-309.

3b) Siehe z. B. H. Freudenthal, Fundam. Math. 29 (1937), 138-144.

4) Die Eigenschaften der Komplexgruppen von Polyedern, von denen wir dabei Gebrauch machen werden, sind: $K$ und $L$ sind freie Gruppen von endlich vielen. Erzeugenden; $\mathfrak{r}$ läßt Division zu (d. $\mathrm{h}$. ist $\mathfrak{x} \alpha t=0 \mathrm{bzw}$. $\mathfrak{x} \alpha l=0$, so ist $\mathfrak{r} k=0$ bzw. $\mathfrak{r} l=0$ oder $\alpha=0$ ). 
Interpretiert man hier die $k_{0}$ bzw. $l_{\emptyset}$ als die $\varrho$-dimensionalen Komplexe von. $R$ bzw. $S$ und $\cdot$ als $\circ$, so hat man genau die Verhältnisse, wie sie bei der Verbindung vorliegen. Interpreticrt man dagegen $k_{\rho}$ bzw. $l_{\rho}$ als die $(\varrho+1)$-dimensionalen Komplexe von $h$ bzw. $S$ und - als $\times$, so liegt alles wie bei der Bildung des Cartesischen Produktes (insbesondere verwandelt sich die Randformel dann in die des Cartesischen Produktes, und an die Stelle der modifizierten treten wieder die gewöhnlichen Bettischen Gruppen $\left.{ }^{5}\right)$ ).

4. Wir gehen nun an den Beweis unserer Formeln, wobei wir uns an das abstrakte Schema halten, also $\cdot$ statt $\circ$ und $\times$ schreiben, aber von den beiden Interpretationen gemeinsamen konkreten Eigenschaften (siehe $\left.{ }^{4}\right)$ ) freizügig Gebrauch machen.

Die Elemente $x_{\rho}$ bzw. $y_{\sigma}$ bzw. $z_{\tau}$ von $K_{\rho}$ bzw. $L_{\sigma}$ bzw. $(K \cdot L)_{r}$, für die $\mathfrak{r} x_{\rho}=0$ bzw. $\mathfrak{r} y_{\sigma}=0 \mathrm{bzw}$. $\mathfrak{r} z_{l}=0$ gilt (Zyklen), bilden die Untergruppe $X_{Q}$ bzw. $Y_{\sigma}$ bzw. $Z_{\tau}$; Zyklen, die, als Elemente der zugehörigen Bettischen Gruppe aufgefaßt, die Ordnung $p$ besitzen, werden $\mathrm{ab}$ und zu durch einen oberen Index $p$ unterschieden: $x^{p}, y^{p}$. Dagegen bedeute $x^{(p)}$ bzw. $y^{(p)}$ einen Komplex mit $\mathfrak{r} x \equiv 0$ bzw. $\mathfrak{r} y \equiv 0 \bmod p($ Zyklus $\bmod p)$. Elemente von $\mathfrak{r} K_{\varrho+1}$ bzw. $\mathfrak{r} L_{\sigma+1}$ werden $r_{0}$ bzw. $s_{\sigma}$ gennant.

Jedes Element von $(K \cdot L)_{\tau}$ läßt sich in der Form

$$
\left.\sum k \cdot l+\sum x \cdot l^{\prime} \quad{ }^{6}\right)
$$

schreiben, in der alle auftretenden $k_{\varrho}$ (für jede Dimension $\varrho$ ) linear unabhängig mod $X_{e}$ sein sollen. Handelt es sich um einen Zyklus $z$, so hat man wegen $\mathrm{x} z=0$

$$
\Sigma k \cdot r l \equiv 0 \quad \bmod X,
$$

also wegen der Unabhängigkeit der $k$

$$
\mathfrak{r} l=0 .
$$

Wir können also schreiben

oder sogar

$$
\begin{gathered}
z=\Sigma k \cdot y+\sum x \cdot l \\
z=\Sigma k \cdot y+\Sigma x \cdot l+\Sigma r \cdot l^{\prime},
\end{gathered}
$$

5ie (-1)-te modifizierte Bettische Gruppe besteht immer allein aus der Null, während die 0-te gewöhnliche Bettische Gruppe stets mindestens eine Erzeugende besitzt. Derartige Unterschiede machen sich bei dem abstrakten Schema natürlich nicht bemerkbar.

$\left.{ }^{6}\right)$ Wo es irgend angängig ist, lassen wir die Indere (einen für die Dimension und einen zur Unterscheidung der einzelnen Linearformen gleicher Dimension) weg. wemn vorausgesetzt wird, daß alle auftretenden $x_{0}$ linear unabhängig $\bmod \mathfrak{i} K_{q+1}$ seien. $\mathfrak{i} z=0$ liefert

also

$$
\sum x \cdot r l \equiv 0 \bmod r K,
$$

$$
x \cdot r l \equiv 0 \quad \bmod r K
$$

(für jedes einzelne Glied der Summe). Ist das $x$ hier ein $x^{p}$, so muß für das zugehörige $l$ gelten: $\imath l \equiv 0 \bmod p$. So erhalten wir die Darstellung

$$
z=\Sigma k \cdot y+\sum x^{p} \cdot y^{(p)}+\Sigma r \cdot l .
$$

Die analoge Betrachtung bei den $y_{\sigma}, \bmod \mathfrak{r} L_{\sigma+1}$, führt weiter $z u$ der Darstellung

$$
z=\Sigma x^{p} \cdot y^{(p)}+\sum x^{(q)} \cdot y^{q}+\sum r \cdot l+\sum k \cdot s .
$$

Auf Grund der Definition von $x^{p}$ und $x^{(q)}$ besitzen die Gleichungen .

$$
\mathfrak{r} \bar{x}^{(p)}=p x^{p}, \quad \mathfrak{r} x^{(q)}=q \bar{x}^{q} .
$$

Lösungen $\bar{x}^{(p)}, \bar{x}^{\prime \prime}$ (im ersten Fall ist die Lösung bis auf ein $x$, im aweiten Fall eindeutig bestimmt); ist $q=0$, so setze man $\bar{x}^{q}=0$. Analog hat man

$$
\mathfrak{r} \bar{y}^{(q)}=q y^{q}, \quad \mathfrak{r} y^{(p)}=p \bar{y}^{p} .
$$

Setzt man Zyklen der Form

$$
\left[x_{0}^{p} y_{\sigma-1}^{p}\right]=x_{Q}^{p} \cdot \bar{y}_{\sigma}^{(p)}-(-1)^{q} \bar{x}_{Q+1}^{(p)} \cdot y_{\sigma-1}^{p}
$$

(wo die gemäß (2) und (3) bestimmten $\bar{x}^{p}, \bar{y}^{p}$ irgendwie festgelegt seien) heraus, so wird aus (1)

$$
z=\sum x \cdot y+\sum_{p \neq 0}\left[x^{p} y^{p}\right]+\sum_{q \neq 0} x^{(q)} \cdot y^{q}+\sum r \cdot l+\sum k \cdot s .
$$

Jedes $s$ ist hier von der Form $s=\mathrm{r} l^{\prime}$. Wir können statt der $l$ und $l^{\prime}$ eine Basis $\left(l^{\prime \prime}\right)$ mod $Y$ zugrundelegen:

$$
z=\ldots+\sum r_{\ell, i} \cdot l_{\sigma, i}^{\prime \prime}+\sum(-1)^{\varrho} k_{\ell+1, i} \cdot \mathrm{r} l_{\sigma, i}^{\prime \prime} .
$$

Berücksichtigt man $\mathrm{r} z=0$, so erhält man wegen der Unabhängigkeit der $l^{\prime \prime}: \quad r_{\varrho, i}=r k_{\varrho}+1, i$, also

$$
z=\sum x \cdot y+\sum_{p \neq 0}\left[x^{p} y^{p}\right]+\sum_{q \neq 0} x^{(q)} \cdot y^{q}+\mathrm{r} m
$$

Hier darf man die auftretenden $y^{q}$ linear unabhängig wählen, und man erhält wegen $\mathfrak{r} z=0: \sum_{q \neq 0} q \bar{x}^{q} \cdot y^{q}=0$, also $\bar{x}^{q}=0$, also

$$
z=\sum x \cdot y+\sum_{p \neq 0}\left[x^{p} y^{p}\right]+\mathrm{r} m .
$$


Man lege jetzt für die $x$ (einschließich der $x^{p}$ ) eine Homologiebasis zugrunde, ebenso für die $y$ (einschließlich der $y^{p}$ ). Dann wird aus (5)

$$
z=\Sigma \alpha(x \cdot y)+\Sigma \beta\left[x^{p} y^{q}\right]+\Sigma x \cdot s+\Sigma y \cdot r+\mathfrak{r} m
$$

mit

$$
\left[x^{p} y^{q}\right]=p^{*}\left(x^{p} \cdot \bar{y}^{(q)}\right)-(-1)^{\varphi} q^{*}\left(x^{(p)} \cdot y^{q}\right) ;
$$

dabei sollen $p^{*}$ und $q^{*}$ teilerfremde Zahlen sein, die sich wie $p$ und $q$ verhalten, $\alpha$ soll alle ganzen Zahlen durchlaufen kzönnen und $\beta$ alle Reste $\bmod (p, q)$ (größter gem. Teiler von $p$ und $q$ ). Man beachte im Folgenden, daß wegen der Unabhängigkeit der $x^{p}$ die $\bar{x}^{(p)}$ unabhängig mod $X$ sind (analog für die $y^{p}$ ).

Sei nun $z$ ein Rand, also

$$
\Sigma \alpha(x \cdot y)+\Sigma \beta\left[x^{p} \cdot y^{q}\right]=\Sigma \mathfrak{r}(k \cdot l)
$$

die rechte Seite läßt sich dann auch schreiben mit mod $X$ unabhängigen $k^{\prime}$ und mod $Y$ unabhängigen $l^{\prime}$ :

$$
\sum\left(\mathfrak{r} k^{\prime} \cdot l^{\prime} \pm k^{\prime} \cdot \mathfrak{r} l^{\prime}\right)+\Sigma x \cdot s+\Sigma r \cdot y \cdot
$$

Vergleich mit der linken Seite liefert

$$
k^{\prime}=\Sigma \gamma \bar{x}^{(p)}, \quad l^{\prime}=\Sigma \delta \bar{y}^{(q)}, \quad r=0, \quad s=0,
$$

so daß die rechte Seite die Gestalt

annimmt.

$$
\sum \varepsilon \cdot\left(p x^{p} \cdot \bar{y}^{(q)} \pm q \bar{x}^{(p)} \cdot y^{q}\right)=\Sigma \varepsilon \cdot(p, q)\left[x^{p} y^{q}\right]
$$

Somit liefern die $x \cdot y$ und $\left[x^{p} y^{q}\right]$ aus (6) eine Basis der Bettischen Gruppe von $K \cdot L$, und da das direkte Produkt einer zyklischen Gruppe der Ordnung $p$ und einer der Ordnung $q$ eine der Ordnung $(p, q)$ ist, ist unsere Formel bewiesen.

\section{Die schlichten stetigen Bilder des Nullraums.}

Von

$$
\text { F. H a us dorff (Bonn). }
$$

Das Folgende ist eine kleine Ergänzung zu Herrn Kuratowski's 1) Theorie der schlichten, beiderseits Borelschen Abbildungen (homéomorphies de classe $\alpha, \beta$ ). Es ist dort festgestellt, dass jede Borelsche Menge $A$ eines separablen vollständigen Raumes schlichtes stetiges Bild einer im Nullraum $N$ abgeschlossenen Menge $N_{0}$ und, falls unabzählbar, nach Tilgung einer abzählbaren Menge $R$ schlichtes stetiges Bild von $N$ selbst ist; ausserdem wird die Klasse der inversen Abbildung präzisiert (vgl. unten (5)). Wenn wir fragen, wann $A$ schlichtes stetiges Bild von $N$ selbst ist (also $N_{0}=N$ oder $R=0$ angenommen werden kann), so ergibt sich als notwendige Bedingung, dass $A$ wie $N$ verdichtet sein muss; dies erweist sich aber auch als binreichend: die schlichten stetigen Bilder von $N$ sind mit den verdichteten Borelschen Mengen identisch ${ }^{2}$ ). Auch hierbei soll die Klasse der inversen Abbildung präzisiert werden (Satz I).

Wir stellen zunächst in (1) bis (7) einige Hilfsbetrachtungen und bekannte Resultate zusammen.

(1) Zur Abkürzung sollen die Borelschen Mengen eines metrischen Raumes nach dem Vorbild von H. Lebesgue so bezeichnet werden:

$$
\begin{aligned}
& F^{0}=F \text { abgeschlossen, } \\
& F^{\alpha}=\Pi_{n} G^{\xi n}
\end{aligned}
$$$$
\begin{aligned}
& G^{0}=G \text { offen, } \\
& G^{\alpha}=\sum F^{\xi_{n}}
\end{aligned}
$$

(für $\left.a>0, \xi_{n}<\alpha\right)$.

1) Wir zitieren die beiden Werke:

(A) Topologie I, Monografie Matematyczne, Warszawa-Lwów 1933.

(B) Sur une généralisation de la notion d'homéomorphie, Fund. Math. 22 (1934), p. 206-220.

2) Vgl. W. Sierpiński, Sur les images continues et biunivoques de l'ensemble de tous les nombres irrationnels, Mathematica 2 (1929), p. 18-21 (für lineare Borelsche Mengen). 